\title{
Purinergic regulation of vascular endothelial growth factor signaling in angiogenesis
}

\author{
SM Rumjahn', N Yokdang', KA Baldwin', J Thai' and ILO Buxton*,I \\ 'Department of Pharmacology, University of Nevada School of Medicine, Reno, NV 89557, USA
}

\begin{abstract}
P2Y purine nucleotide receptors (P2YRs) promote endothelial cell tubulogenesis through breast cancer cell-secreted nucleoside diphosphate kinase (NDPK). We tested the hypothesis that activated P2Y, receptors transactivate vascular endothelial growth factor receptor (VEGFR-2) in angiogenic signaling. P2Y, R stimulation ( $10 \mu \mathrm{M}$ 2-methyl-thio-ATP (2MS-ATP)) of angiogenesis is suppressed by the VEGFR-2 tyrosine kinase inhibitor, SUI 498 (I $\mu \mathrm{M}$ ). Phosphorylation of VEGFR-2 by 0.0262 or 2.62 nM VEGF was comparable with 0.01 or $10 \mu \mathrm{M}$ 2MS-ATP stimulation of the P2Y,R. 2MS-ATP, and VEGF stimulation increased tyrosine phosphorylation at tyrl 175. 2MS-ATP $(0.1-10 \mu \mathrm{M})$ also stimulated EC tubulogenesis in a dose-dependent manner. The addition of sub-maximal VEGF $(70 \mathrm{PM})$ in the presence of increasing concentrations of 2MS-ATP yielded additive effects at 2MS-ATP concentrations $<3 \mu \mathrm{M}$, whereas producing saturated and less than additive effects at $\geqslant 3 \mu \mathrm{M}$. We propose that the VEGF receptor can be activated in the absence of VEGF, and that the P2YR-VEGFR2 interaction and resulting signal transduction is a critical determinant of vascular homoeostasis and tumour-mediated angiogenesis.
\end{abstract}

British Journal of Cancer (2009) I 00, I465 - |470. doi: I0.1038/sj.bjc.6604998 www.bjcancer.com

Published online 14 April 2009

(C) 2009 Cancer Research UK

Keywords: breast cancer; angiogenesis; purinergic receptor; P2Y; VEGF; VEGFR2; phosphotyrosine

The secretion of nucleoside diphosphate kinase (NDPK; EC 2.7.4.6) orthologues by intracellular parasites (Gounaris et al, 2001; Chopra et al, 2003), NDPK secretion by various carcinomas (Anzinger et al, 2001; Okabe-Kado and Kasukabe, 2003), and NDPK's extracellular role in blood flow regulation (Buxton et al, 2001) first lead us to propose a pathological role for secreted NDPK in cancer and tumour angiogenesis. We have recently provided evidence for a nucleotide-dependent regulation of angiogenesis by breast cancer-secreted NDPK (Rumjahn et al, 2007). We observed that extracellular NDPK by regeneration of extracellular nucleotides can use endothelial P2 (Y) nucleotide receptors to stimulate angiogenesis. Supporting our findings, the disruption of CD39 (ecto-apyrase EC 3.6.1.5) activity, the dominant vascular ectonucleotidase and its regulation of nucleotide signaling, has been observed to inhibit tumour angiogenesis and metastasis (Goepfert et al, 2001; Jackson et al, 2007). The regulation of extracellular ATP and ADP levels by ecto-apyrase is also known to play important roles in cardiovascular physiology and pathophysiology by activation of purinergic type-2 (P2) nucleotide receptors (Erlinge and Burnstock, 2008).

P2 nucleotide receptors activated by ATP include both ligandgated ion channels (P2X) and heterotrimeric G protein-coupled receptors $(\mathrm{P} 2 \mathrm{Y})$. $\mathrm{P} 2 \mathrm{Y}$ receptors have become recognised as the important regulators of carcinogenesis, endothelial regulation, and blood flow regulation (Buxton et al, 2001; Burnstock, 2006; White

*Correspondence: Professor ILO Buxton, Department of Pharmacology MS3 18, University of Nevada School of Medicine, 1664 N Virginia St., Reno, NV 89557, USA; E-mail: ibuxton@medicine.nevada.edu

Received 25 November 2008; revised 29 January 2009; accepted 26 February 2009; published online 14 April 2009 and Burnstock, 2006). Little is known about the role of P2Y receptors (P2YRs) in angiogenesis with only a handful of reports providing evidence supporting this notion. We have shown earlier that P2YR signaling promotes angiogenic properties such as endothelial cell tubulogenesis (Rumjahn et al, 2007), whereas others have reported P2YR-mediated migration (Satterwhite et al, 1999; Kaczmarek et al, 2005) and permeability (Tanaka et al, 2004, 2006). Activated $\mathrm{P}_{2} \mathrm{Y}_{2}$ receptors can transactivate vascular endothelial growth factor receptor-2 (VEGFR-2), suggesting a direct link between extracellular nucleotides and established tumour angiogenesis signaling (Seye et al, 2004). VEGFR-2 mediates the majority of the angiogenic and permeabilityenhancing effects of VEGF (Shibuya, 2006). Given this evidence, we hypothesised that endothelial P2YR signaling interacts to regulate VEGFR-2 signaling. Here, we provide evidence that $\mathrm{P} 2 \mathrm{Y}_{1} \mathrm{R}$ stimulation of human endothelial cells activates VEGFR-2 intracellular signaling to stimulate endothelial cell tubulogenesis, a direct in vitro measure of angiogenesis. These data suggest that tumour-mediated angiogenesis signaling may be, in part, mediated by nucleotide receptor activation of the VEGFR-2 pathway and may effectively lower the local requirement for VEGF.

\section{MATERIALS AND METHODS}

\section{Cell culture}

Human cardiac endothelial cells (HCECs) were earlier isolated by Fluorescence Activated Cell Sorting for CD31 (PECAM) and immortalised by human telomerase reverse transcriptase (hTRT). Cloned human cord blood endothelial colony forming cells (ECFCs) were purchased from Dynacell Life Sciences (Spring 
House, PA, USA) and used experimentally between passages 6 and 13. The HCEC population represents an immortalised cell line, whereas the ECFC population represents a primary cell line.

Human cardiac endothelial cells were grown in Dulbecco's Modified Eagle's Medium (HyClone, Logan, UT, USA) supplemented with $10 \%$ foetal bovine serum ((FBS) Atlanta Biological, Lawrenceville, GA, USA), penicillin-streptomycin $\left(1500 \mathrm{Ul}^{-1}\right.$ $-100 \mathrm{mgl}^{-1}$ ), and $0.5 \mathrm{mgl}^{-1}$ fungizone (Invitrogen, Carlsbad, CA, USA). Human ECFCs were grown in endothelial growth media-2 ((EGM-2) Clonetics, East Rutherford, NJ, USA) supplemented with $10 \% \mathrm{FBS}(\mathrm{v} / \mathrm{v})$, penicillin - streptomycin, and fungizone as above. EGM-2 without angiogenic growth factors (EBM-2, Clonetics) and with low serum (2\% FBS) was used during angiogenic experimental treatments. Cells were grown and maintained at $37^{\circ} \mathrm{C}$ in a humidified atmosphere with $5 \% \mathrm{CO}_{2} / 95 \%$ air.

\section{In vitro angiogenesis scoring technique}

A representative endothelial cell tubulogenesis (angiogenesis) score for each condition was obtained by analysing digital images $(\times 100)$ collected from the central pointing corners of quadrants I-IV in each culture well and averaging the four scores. As described earlier (Rumjahn et al, 2007), an angiogenesis score $(s)$ represents the product of mean number of branch points $(b p)$ multiplied by mean pixel length $(l)$ multiplied by mean pixel cell surface area $(a)$. Thus, $s=b p \times l \times a$.

\section{Effect of disrupting VEGF signaling in P2Y receptor-mediated angiogenesis}

Human cardiac endothelial cells $\left(3 \times 10^{4}\right)$ on collagen-coated plates were incubated for $24 \mathrm{~h}$ with $\mathrm{P} 2 \mathrm{Y}$ receptor agonists ATP $\left(\mathrm{P}_{1 / 2} \mathrm{Y}_{1 / 2} \mathrm{R} ; 100 \mu \mathrm{M}\right)$ and 2-methyl-thio-ATP (2MS-ATP) (P2Y ${ }_{1} \mathrm{R}$; $10 \mu \mathrm{M})$. EC tubulogenesis was also observed in the presence of $1 \mu \mathrm{M}$ SU1498 ( $\sim \mathrm{IC}_{50}$ of VEGFR-2 tyrosine kinase inhibitor (Strawn et al, 1996); Sigma, St Louis, MO, USA) with either $100 \mu \mathrm{M}$ ATP or $10 \mu \mathrm{M}$ 2MS-ATP. EGM-2 was employed as a positive control, whereas non-treatment controls were performed for normalisation and comparison. EGM-2 stimulated growth was also observed in the presence of $1 \mu \mathrm{M}$ SU1498 as a VEGFR-2 inhibition control. The use of $10 \mu \mathrm{M}$ 2MS-ATP or $100 \mu \mathrm{M}$ ATP in promoting EC tubulogenesis was maximal, consistent with known desensitisation of $\mathrm{P} 2 \mathrm{Y}_{1 / 2}$ receptors at higher agonist concentrations (Ralevic and Burnstock, 1998; Rumjahn et al, 2007). SU1498 was chosen as it is at least 100 -fold more selective for VEGFR-2 kinase compared with other receptor tyrosine kinases such as platelet-derived growth factor receptor, epidermal growth factor (EGF) receptor, and human epidermal growth factor receptor 2 (Strawn et al, 1996).

Endothelial colony forming cells $\left(5 \times 10^{4}\right)$ plated on growth factor reduced (GFR) Matrigel (BD Biosciences, Bedford, MA, USA) were incubated for $24 \mathrm{~h}$ with varying concentrations $(0.05-$ $10 \mathrm{ng} / \mathrm{ml} ; 1.31$ - $262 \mathrm{pM}$ ) of VEGF-A (VEGF; Sigma). Non-treatment controls were performed for normalisation and comparison. The experimentally determined maximal VEGF stimulation of EC tubulogenesis (262 pM) was used as a positive control in all subsequent P2Y/VEGF experiments.

Endothelial colony forming cells $\left(5 \times 10^{4}\right)$ grown on GFR Matrigel were incubated for $24 \mathrm{~h}$ in the presence of $10 \mu \mathrm{M} 2 \mathrm{MS}$ ATP with or without $1 \mu \mathrm{M}$ SU1498. VEGF (262 pM) in EBM-2 was used as a positive control and was also used in the presence of $1 \mu \mathrm{M}$ SU1498 to show the VEGFR-2-specific promotion of EC tubulogenesis. Non-treatment controls were performed for normalisation and comparison.

\section{Effect of $\mathrm{P}_{2} \mathrm{Y}_{1}$ receptor activation on VEGFR-2 activation}

Endothelial colony forming cells were first grown to $\sim 75 \%$ confleuncy on collagen-coated plates and then switched to EBM-2 supplemented with $0.5 \%$ FBS (v/v) overnight. ECFCs were next stimulated with either VEGF $(0.0262$ or $2.62 \mathrm{nM})$ or $2 \mathrm{MS}$-ATP $(10 \mathrm{nM}$ or $10 \mu \mathrm{M})$ for $10 \mathrm{~min}$. The cells were collected in cold NP-40 lysis buffer. This cell lysate was centrifuged at $10000 \mathrm{~g}$ to remove cellular debris.

Cell lysate supernatants $(150 \mu \mathrm{g})$ were incubated with KDR/ VEGFR-2 specific antibody $(0.3 \mu \mathrm{g}$ rabbit IgG; Chemicon, Rockford, IL, USA) and protein A/G agarose beads ( $20 \mu$; Santa Cruz Biotechnology Inc., Santa Cruz, CA, USA). Proteins were separated by electrophoresis on $10 \%$ polyacrylamide gels (Bio-Rad, Hercules, CA, USA) and transferred to nitrocellulose membranes. The membranes were then labeled for either overall phosphotyrosines (1:1000 mouse IgG; Chemicon) or VEGFR-2 phosphotyrosine 1175 (1:500 rabbit IgG; Cell Signaling Technology, Danvers, MA, USA). Respective secondary antibodies conjugated to either Alexa Fluor 680 or Alexa Fluor 800 fluorescent dye (1:100000; Invitrogen) were used for detection. Antibody incubations were carried out in 1:1 Odyssey blocking buffer (Licor Biosciences, Lincoln, NE, USA) and PBS with $0.1 \%$ Tween-20 (v/v). Bands were visualised using the Odyssey Infrared Imaging System (V2.04). VEGFR-2 levels were checked for equal loading.

\section{Effect of 2-Methyl-Thio-ATP signaling on angiogenesis}

Endothelial colony forming cells $\left(5 \times 10^{4}\right)$ on GFR Matrigel-coated plates were incubated for $24 \mathrm{~h}$ with various concentrations of 2MS-ATP (0.1-10 $\mu \mathrm{M}$ in half-log increments). VEGF (262 pM) was used as a positive control, whereas non-treatment controls were performed for normalisation and comparison.

\section{Effect of 2-Methyl-Thio-ATP and vascular endothelial growth factor signaling on angiogenesis}

Endothelial colony forming cells $\left(5 \times 10^{4}\right)$ on GFR Matrigel-coated plates were incubated for $24 \mathrm{~h}$ with various concentrations of 2MS-ATP $(0.1-10 \mu \mathrm{M}$ in half-log increments) in the presence of sub-maximal VEGF stimulation (ECFC tubulogenesis $\sim \mathrm{EC}_{50}$ as experimentally determined; $70 \mathrm{pM}$ ) in order to determine potential co-activation between these angiogenic molecules. VEGF (262 pM) was used as a positive control, whereas non-treatment controls provided normalisation and comparison.

\section{Statistical analyses}

All graphs were prepared using Prism Graphing Software (V5.01; GraphPad Software, San Diego, CA, USA) and statistical analyses were carried out using InStat Statistical Software (V3.06; GraphPad Software), with $P \leqslant 0.05$ considered significant. Significance was determined using non-parametric analysis of variance (KruskalWallis) with Dunn's multiple comparisons post-test or nonparametric $t$-test (Mann-Whitney). Data points and error bars represent means \pm s.e.m. ${ }^{\star} P \leqslant 0.05 ;{ }^{*} P \leqslant 0.01 ;{ }^{* * *} P \leqslant 0.001$ (vs negative control, unless otherwise indicated); ${ }^{\#} P \leqslant 0.05$ (2MS-ATP alone $v s$ 2MS-ATP + VEGF combination).

\section{RESULTS}

\section{Endothelial $\mathrm{P} 2 \mathrm{Y}_{1 / 2}$ receptors mediate in vitro angiogenesis through VEGF signaling}

As P2Y receptors have earlier been reported to interact with VEGFR-2 (Seye et al, 2004), we investigated whether nucleotides and VEGF-induced angiogenesis involved a common signaling pathway. HCECs incubated with $\mathrm{P}_{2} \mathrm{Y}_{1 / 2}$ receptor agonists $(100 \mu \mathrm{M}$ ATP or $10 \mu \mathrm{M} 2 \mathrm{MS}$-ATP) for $24 \mathrm{~h}$ showed a $\sim 2.0$-fold angiogenic stimulation $(P \leqslant 0.01$; Figure $1 \mathrm{~A})$ that was suppressed back to $\sim 1.3$-fold control by $1 \mu \mathrm{M}$ SU1498. The $\sim \mathrm{IC}_{50}$ of SU1498, a VEGFR-2 tyrosine kinase inhibitor, was used to limit non-specific 


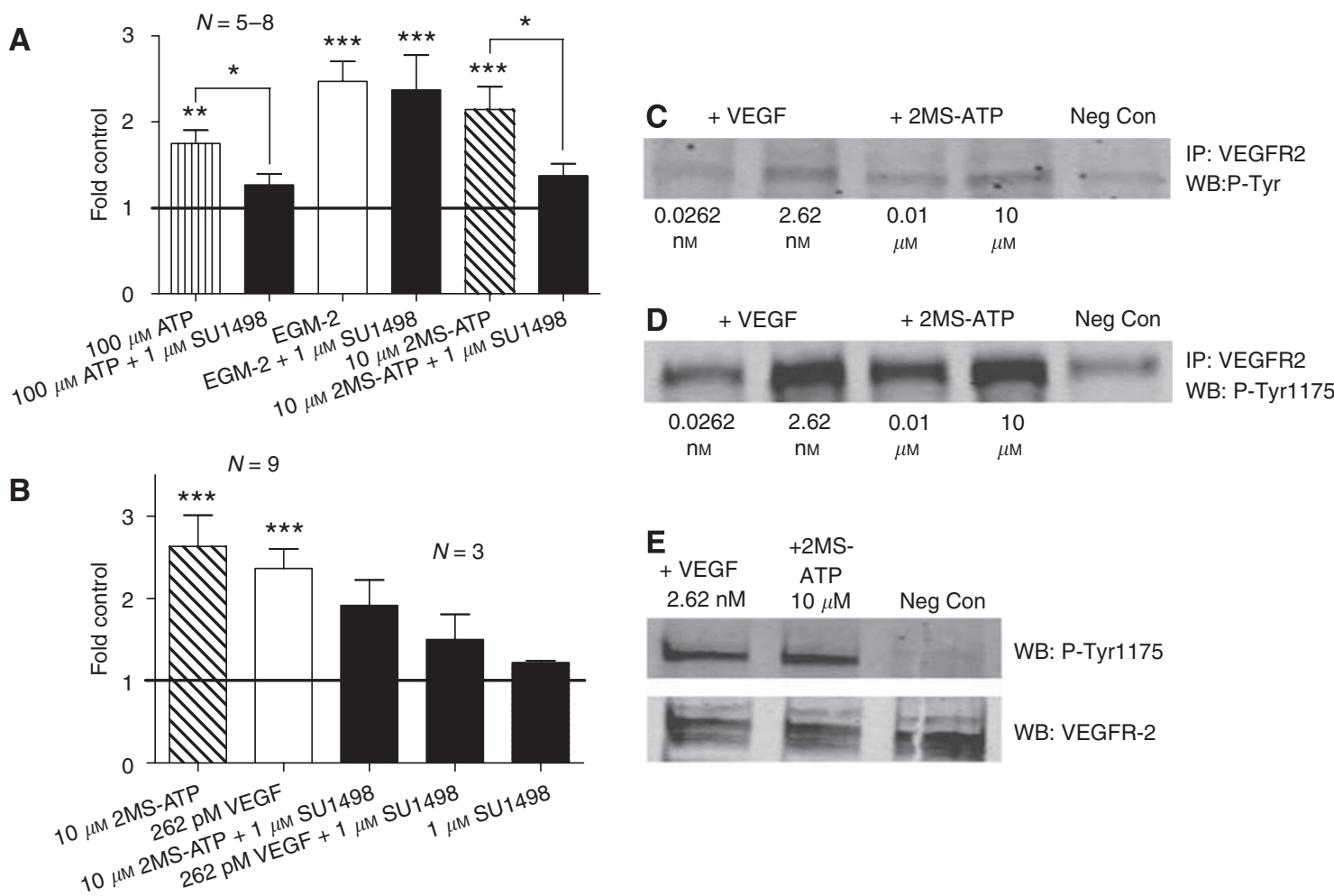

Figure I Endothelial P2Y receptor-mediated in vitro angiogenesis uses vascular endothelial growth factor (VEGF) signaling. Inhibition of VEGFR-2 intracellular signaling by SUI 498 suppressed the pro-angiogenic potential of P2Y,/2 receptor agonists ATP and/or 2-methyl-thio-ATP (2MS-ATP) during a $24 \mathrm{~h}$ EC tubulogenesis assay. (A) Control mean $=979.4 \pm 403.6$ angiogenesis units. Negative control $\mathbf{A}$; HCECs incubated in CDMEM supplemented with $2 \% \mathrm{FBS}$ and $0.01 \%(\mathrm{v} / \mathrm{v})$ DMSO. The angiogenic stimulation control used was endothelial growth media-2 (EGM-2). (B) Control mean $=817.4 \pm 31.1$ angiogenesis units. Negative control B; ECFCs incubated in EBM-2 supplemented with $2 \% \mathrm{FBS}$ and $0.01 \%$ (v/v) DMSO. The angiogenic stimulation control used was EBM-2 containing VEGF, which was also suppressed by SUI498. (C-E); ECFCs treated with either VEGF (natural VEFGR-2 agonist) or 2MS-ATP (P2Y, R agonist) for $10 \mathrm{~min}$ observed respective phosphorylation and trans-phosphorylation of VEGFR-2. Samples were immunoprecipitated for VEGFR-2 and western blotted for $(\mathbf{C})$ overall phosphotyrosines or (D) phosphotyrosine II75. (E) Samples were directly western blotted for VEGFR-2 and phosphotyrosine I 175. Negative control C and D; ECFCs incubated in EBM-2 supplemented with 0.5\% FBS. Negative control E; EBM-2 with I\% FBS. $* P \leqslant 0.05 ; * * P \leqslant 0.01 ; * * * * \leqslant 0.001$.

inhibition of other RTKs (e.g., platelet-derived growth factor receptor and epidermal growth factor receptor) as reported earlier with higher concentrations of this agent (Strawn et al, 1996). The EGM-2 control produced a $\sim 2.5$-fold increase over control $(P \leqslant 0.001$; Figure 2), which, interestingly, was not affected by the addition of $1 \mu \mathrm{M}$ SU1498. EGM-2 (a proprietary cocktail) includes a mix of various angiogenic factors, including VEGF.

Using primary human endothelial cells, we also observed that $\mathrm{P}_{2} \mathrm{Y}_{1}$ receptor activation stimulates VEGFR-2-mediated EC tubulogenesis. ECFCs incubated with $10 \mu \mathrm{M}$ 2MS-ATP $\left(\mathrm{P}_{2} \mathrm{Y}_{1}\right.$ receptor agonist) for $24 \mathrm{~h}$ exhibited a $\sim 2.6$-fold angiogenic stimulation $(P \leqslant 0.001$; Figure $1 \mathrm{~B})$ that was suppressed back to $\sim 1.9$-fold control by $1 \mu \mathrm{M}$ SU1498. The VEGF control (262 pM) observed a $\sim 2.4$-fold increase over control, which was suppressed back to $\sim 1.5$-fold control by $1 \mu \mathrm{M}$ SU1498. The SU1498 control showed minimal angiogenic effect alone.

\section{Activated endothelial $\mathrm{P}_{2} \mathrm{Y}_{1}$ receptors transphosphorylate VEGFR-2}

It is well known that ligand binding of a RTK (e.g., VEGFR-2) induces dimerisation and subsequent activation of the receptor, resulting in the autophosphorylation of tyrosine residues in its cytoplasmic domain (Arora and Scholar, 2005). Knowing that P2 $\mathrm{Y}_{1} \mathrm{R}$-mediated EC tubulogenesis uses VEGFR-2 intracellular signaling, we further asked if $\mathrm{P} 2 \mathrm{Y}_{1} \mathrm{R}$ activation by $2 \mathrm{MS}$-ATP would transphosphorylate (i.e., transactivate) VEGFR-2. ECFCs stimulated with VEGF (0.0262 or $2.62 \mathrm{~nm}$ ) showed phosphorylation of
VEGFR-2 in a dose-dependent manner (Figure 1C; lanes 1 and 2), consistent with the known activation of this receptor by its natural ligand. ECFCs stimulated with 2MS-ATP $(0.01$ and $10 \mu \mathrm{M})$ also exhibited a similar dose-dependent phosphorylation of VEGFR-2 (Figure 1C; lanes 3 and 4). The non-stimulated (negative) control showed a minimal level of basal VEGFR-2 tyrosine phosphorylation (Figure 1C; lane 5).

Phosphorylated tyrosine 1175 of VEGFR-2 is a binding site for the $\mathrm{SH} 2$ domain of phospholipase $\mathrm{C}$, which is an important mediator of VEGFR-2-induced angiogenesis (Shibuya, 2006). We further observed that ECFCs stimulated with either VEGF or 2MS-ATP phosphorylated VEGFR-2 Tyr1175 in a similar dose-dependent fashion (Figure 1D and E). Human umbilical vein endothelial cells also showed similar levels of VEGFR-2 tyrosine phosphorylation by VEGF and 2MS-ATP (data not shown).

\section{Endothelial $\mathrm{P}_{2} \mathrm{Y}_{1}$ receptor activation stimulates EC tubulogenesis in a dose-dependent manner}

We observed that 2MS-ATP promotes a dose-dependent angiogenic response with significant stimulation seen at higher concentrations of $\geqslant 3 \mu \mathrm{M}$ 2MS-ATP, $\sim 2.5$-fold increase over control $(P \leqslant 0.05$; Figure $2 \mathrm{~A})$. We observed earlier that $\geqslant 10 \mu \mathrm{M}$ 2MS-ATP does not provide additional angiogenic stimulation (Rumjahn et al, 2007), therefore an apparent tubulogenesis $\mathrm{EC}_{50}$ would be $\sim 3 \mu \mathrm{M}$. The VEGF control (262 pM) stimulated angiogenesis similar to that seen with $10 \mu \mathrm{M} 2 \mathrm{MS}-\mathrm{ATP}$. 

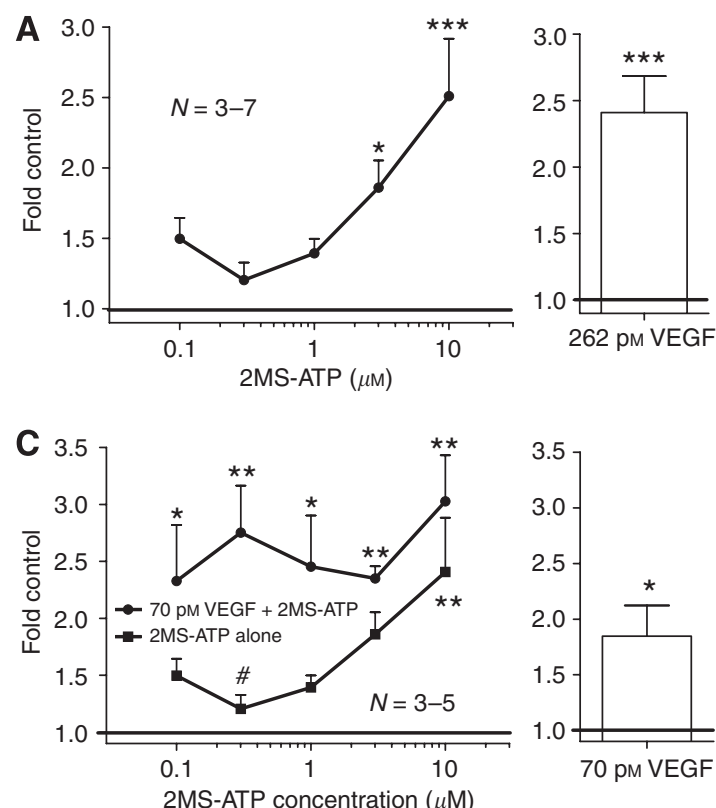

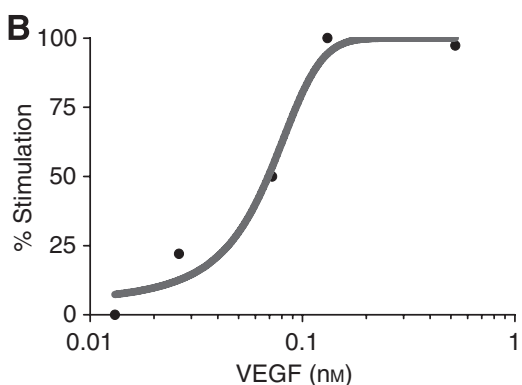

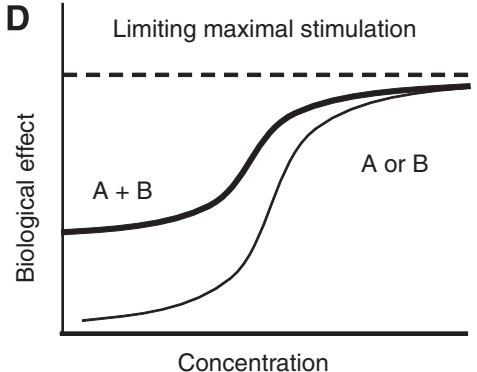

Figure 2 2-methyl-thio-ATP (2MS-ATP) and vascular endothelial growth factor (VEGF) cooperatively promote in vitro angiogenesis. P2Y, or VEGF signaling alone, as well as together stimulated EC tubulogenesis over a $24 \mathrm{~h}$ duration. (A) ECFCs treated with varying amounts of 2 MS-ATP produced a dose-dependent stimulation of tubulogenesis. Control mean $=1292.8 \pm 65.1$ angiogenesis units. Negative control A; ECFCs incubated in EBM-2 supplemented with $2 \%$ FBS. The angiogenic stimulation control used was EBM-2 containing VEGF. (B) VEGF (natural VEFGR-2 agonist) produced a dosedependent stimulation of tubulogenesis. Angiogenic responses varied between I.25- and 3.00-fold control (defined as 0 and I00\% stimulation). Negative control B; ECFCs incubated in EBM-2 supplemented with 2\% FBS. Curve trace was calculated using a non-linear fit of the data employing an equation describing a sigmoidal curve. (C) ECFCs incubated with varying concentrations of 2MS-ATP combined with a constant sub-maximal level of VEGF (apparent tubulogenesis $\mathrm{EC}_{50}$ of $70 \mathrm{pM}$ ) produced additive stimulation of $\mathrm{EC}$ tubulogenesis only at lower concentrations of 2MS-ATP. Control mean $=655.7 \pm 81.8$ angiogenesis units. Negative control C; ECFCs incubated in EBM-2 supplemented with 2\% FBS. Fold control - I.00 equals non-stimulated (negative) control. (D) Hypothetical curve illustrating two molecules promoting a biological response (e.g., angiogenesis) via convergent signaling pathways, which limits the potential of a larger response at higher concentrations. $* P \leqslant 0.05 ; * * P \leqslant 0.01 ; * * * P \leqslant 0.001$.

\section{VEGF stimulates in vitro angiogenesis in a dose-dependent manner}

Endothelial colony forming cells incubated with varying concentrations of VEGF $(0.00131-0.524 \mathrm{nM})$ over $24 \mathrm{~h}$ also exhibited a dose-dependent increase in EC tubulogenesis with maximal response at levels $\geqslant \sim 0.262 \mathrm{~nm}$ (Figure $2 \mathrm{~B}$ ). On the basis of these observations, an apparent VEGF $\mathrm{EC}_{50}$ of $\sim 70 \mathrm{pm}$ was used as a sub-optimal level of VEGF stimulation of tubulogenesis.

\section{MS-ATP and VEGF use a common angiogenic pathway}

To further investigate the notion of P2Y and VEGF cooperative signaling, we incubated ECFCs with varying concentrations of 2MS-ATP $(0.1-10 \mu \mathrm{M})$ in the presence of constant tubulogenesis $\mathrm{EC}_{50}$ concentration for VEGF (70 pM). We observed that the combination of sub-optimal levels of 2MS-ATP and VEGF do indeed promote a significant dose-dependent angiogenic response when compared with 2MS-ATP alone and control $(P \leqslant 0.05$; Figure 2C). Lower concentrations of 2MS-ATP $(<3 \mu \mathrm{M})$ in combination with VEGF (70 pM) exhibited additive-like angiogenic stimulation, whereas higher concentrations ( $\geqslant 3 \mu \mathrm{M} 2 \mathrm{MS}-\mathrm{ATP})$ produced a more saturated and less than additive promotion of EC tubulogenesis. VEGF at $70 \mathrm{pM}$ provided $\sim 51 \%$ of maximal VEGF stimulated EC tubulogenesis (compared with $262 \mathrm{pM}$ ), consistent with our preliminary determination of the $\mathrm{EC}_{50}$.

\section{DISCUSSION}

We observed earlier that pathologically secreted NDPK stimulates angiogenesis in a nucleotide-dependent manner principally by $\mathrm{P}_{2} \mathrm{Y}_{1} \mathrm{R}$ (Rumjahn et al, 2007). Supporting this notion of
P2Y-mediated angiogenesis, it has been reported that endothelial $\mathrm{P}_{2} \mathrm{Y}_{2} \mathrm{R}$-mediated VEGFR-2 activation stimulates the expression of pro-inflammatory vascular cell adhesion molecule 1 (VCAM-1) (Seye et al, 2003, 2004). Inflammation and angiogenesis share common mechanisms and are often seen concurrently, especially in carcinogenesis. An appropriate example would be the use of VCAM-1 in the recruitment of monocytes (undifferentiated macrophages) and the differentiation of these cells into tumourassociated macrophages, which can provide a microenvironment conducive to tumour growth, metastasis, and of course angiogenesis, as especially prevalent in breast and prostate cancers (Pollard, 2004).

We suggest that P2YR-mediated VEGFR-2 activation can promote tumour angiogenesis indirectly, as well as stimulate direct angiogenic effects on the endothelial cells where the original P2Y/VEGF signaling occurs. We investigated the latter notion and observed the inhibition of ATP and 2MS-ATP-mediated EC tubulogenesis by VEGFR-2 tyrosine kinase inhibitor SU1498. This showed that VEGFR-2 intracellular signaling is a substantial component of P2YR-mediated in vitro angiogenesis. The unaffected angiogenic potential of EGM-2 (includes angiogenic factors in addition to VEGF) by SU1498 is consistent with the presence of multiple angiogenic pathways that can compensate for impaired VEGF signaling. As this P2Y-mediated VEGFR-2 signaling was observed in immortalised endothelial cells (HCECs), we further showed that this signaling also exists in primary endothelial cells (ECFCs). In the presence of a more relevant VEGF angiogenic control, SU1498 ( $\sim \mathrm{IC}_{50}$ tyrosine kinase activity) produced a $\sim 60 \%$ reduction in stimulated EC tubulogenesis indicating a tight association between VEGFR-2 tyrosine kinase activity and our detection of EC tubulogenesis. VEGF (0.262 nM) exhibited maximal induction, whereas higher concentrations 
( $>1.31 \mathrm{~nm}$ VEGF) exhibited signs of desensitisation, consistent with known internalisation and degradation of activated VEGFR-2 (Ewan et al, 2006).

In addition to our functional angiogenesis data we observed that $\mathrm{P}_{2} \mathrm{Y}_{1} \mathrm{R}$ activation by $2 \mathrm{MS}$-ATP induces tyrosine phosphorylation, general and tyrosine 1175-specific, on the cytoplasmic domain of VEGFR-2 similar to that seen by its natural ligand VEGF. Tyr1175 of VEGFR-2 and its phosphorylation have been shown to be the important regulators of VEGF-dependent angiogenic signaling (Holmqvist et al, 2004; Sakurai et al, 2005; Shibuya, 2006). This P2YR-mediated transphosphorylation and transactivation of VEGFR-2 provides further evidence that P2YR uses VEGF signaling to stimulate angiogenesis. This is the first report of $\mathrm{P}_{2} \mathrm{Y}_{1} \mathrm{R}$ activation of VEGFR-2 angiogenic signaling.

$\mathrm{P} 2 \mathrm{Y}_{1} \mathrm{R}$ activation of VEGFR-2 signaling was further investigated by showing that varying levels of $P 2 Y_{1} R$ activation produces a dose-dependent angiogenic response. When sub-maximal VEGF signaling (experimentally determined $\mathrm{EC}_{50}$ of $\sim 70 \mathrm{pM}$ ) was subtracted from the combination of 2MS-ATP and VEGF, concentrations of $2 \mathrm{MS}-\mathrm{ATP} \geqslant 3 \mu \mathrm{M}$ observed angiogenesis levels less than that seen from 2MS-ATP treatment alone (less than additive effect). This can be conceptually explained when considering the stimulation of a biological response (e.g., EC tubulogenesis) by two molecules using the same signaling pathway, as illustrated in Figure 2D. This dose-dependent saturation of VEGF angiogenic signaling by $2 \mathrm{MS}$-ATP further suggests that $\mathrm{P}_{2} \mathrm{Y}_{1} \mathrm{R}$-mediated angiogenesis is dependent on VEGFR-2 signaling and moreover, that VEGFR-2 does not need its natural ligand to become activated. P2Y receptors may additionally use their own separate signaling pathways independent of VEGFR-2 to promote angiogenesis, and it is possible that these independent pathways converge at one or more distal effectors.

We now further expand our original hypothesis to include VEGFR-2 signaling as a significant contributor to P2YR-induced angiogenesis, which cancer cells can exploit to pathological advantage by secretion of NDPK (Figure 3). Our demonstration of productive interaction between P2Y and VEGF signaling predicts that low levels of ATP and VEGF can cooperate to stimulate considerable angiogenesis. Dynamic regulation of these two molecules (ATP $>$ VEGF or VEGF $>$ ATP) may be used to produce similar angiogenic responses in a temporal- and environment-dependent manner. With respect to pathological angiogenesis, tumour vasculature is more sensitive to VEGF withdrawal as it mimics immature vessels that are extremely dependent on VEGF signaling (Benjamin et al, 1999). Thus we

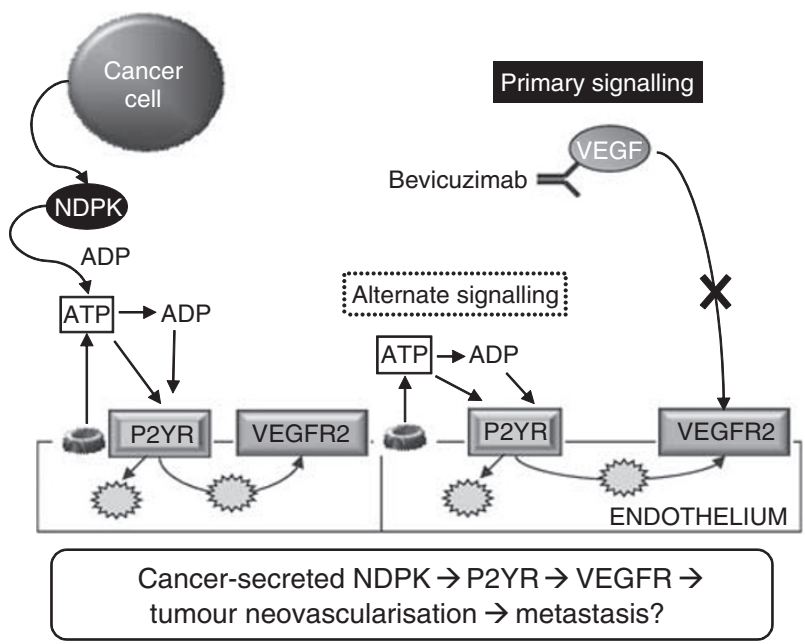

Figure 3 Putative role of extracellular nucleoside diphosphate kinase (NDPK) and P2Y receptor (P2YR)/vascular endothelial growth factor receptor (VEGFR-2) activation in angiogenesis. We have observed breast cancer-secreted NDPK-B to be a significant contributor in promoting angiogenesis. Extracellular NDPK would modulate nucleotides such as elevating ATP levels (Rumjahn et al, 2007). Our current data supports a scenario where P2Y purinergic receptor activation above an unknown threshold would produce conditions favourable to pathological angiogenesis. Moreover, this P2Y angiogenic signaling would cooperate with VEGF angiogenic signal. This posits the notion of dual inhibition of VEGF signaling through sequestering extracellular VEGF levels (i.e., Bevicuzimab) as well as blocking P2YR-dependent activation of VEGFR-2.

propose that dual inhibition of P2YR and VEGFR-2 signaling may provide an effective mode of combinational anti-angiogenic therapy. It is interesting that, this P2YR activation of VEGFR-2 intracellular signaling may also, in part, explain constitutive VEGFR-2 activation in the absence of exogenous VEGF (Lee et al, 2007). Therefore, P2YR-VEGFR2 signaling may be important in describing and understanding VEGF signaling required for endothelial homoeostasis in both tumour as well as normal vasculature.

\section{ACKNOWLEDGEMENTS}

This work was supported by NIH HD053028 and CA09563.

\section{REFERENCES}

Anzinger J, Malmquist NA, Gould J, Buxton IL (2001) Secretion of a nucleoside diphosphate kinase (Nm23-H2) by cells from human breast, colon, pancreas and lung tumors. Proc West Pharmacol Soc 44: 61-63

Arora A, Scholar EM (2005) Role of tyrosine kinase inhibitors in cancer therapy. J Pharmacol Exp Ther 315(3): 971-979

Benjamin LE, Golijanin D, Itin A, Pode D, Keshet E (1999) Selective ablation of immature blood vessels in established human tumors follows vascular endothelial growth factor withdrawal. J Clin Invest 103(2): $159-165$

Burnstock G (2006) Pathophysiology and therapeutic potential of purinergic signaling. Pharmacol Rev 58(1): 58-86

Buxton IL, Kaiser RA, Oxhorn BC, Cheek DJ (2001) Evidence supporting the Nucleotide Axis Hypothesis: ATP release and metabolism by coronary endothelium. Am J Physiol Heart Circ Physiol 281(4): H1657-H1666

Chopra P, Singh A, Koul A, Ramachandran S, Drlica K, Tyagi AK, Singh Y (2003) Cytotoxic activity of nucleoside diphosphate kinase secreted from Mycobacterium tuberculosis. Eur J Biochem 270(4): 625-634

Erlinge D, Burnstock G (2008) P2 receptors in cardiovascular regulation and disease. Purinergic Signal 4(1): 1-20
Ewan LC, Jopling HM, Jia H, Mittar S, Bagherzadeh A, Howell GJ, Walker JH, Zachary IC, Ponnambalam S (2006) Intrinsic tyrosine kinase activity is required for vascular endothelial growth factor receptor 2 ubiquitination, sorting and degradation in endothelial cells. Traffic 7(9): 1270-1282 Goepfert C, Sundberg C, Sevigny J, Enjyoji K, Hoshi T, Csizmadia E, Robson S (2001) Disordered cellular migration and angiogenesis in cd39null mice. Circulation 104(25): 3109-3115

Gounaris K, Thomas S, Najarro P, Selkirk ME (2001) Secreted variant of nucleoside diphosphate kinase from the intracellular parasitic nematode Trichinella spiralis. Infect Immun 69(6): 3658-3662

Holmqvist K, Cross MJ, Rolny C, Hagerkvist R, Rahimi N, Matsumoto T, Claesson-Welsh L, Welsh M (2004) The adaptor protein shb binds to tyrosine 1175 in vascular endothelial growth factor (VEGF) receptor-2 and regulates VEGF-dependent cellular migration. J Biol Chem 279(21): $22267-22275$

Jackson SW, Hoshi T, Wu Y, Sun X, Enjyoji K, Cszimadia E, Sundberg C, Robson SC (2007) Disordered purinergic signaling inhibits pathological angiogenesis in cd39/Entpd1-null mice. Am J Pathol 171(4): 1395- 1404 Kaczmarek E, Erb L, Koziak K, Jarzyna R, Wink MR, Guckelberger O, Blusztajn JK, Trinkaus-Randall V, Weisman GA, Robson SC (2005) 
Modulation of endothelial cell migration by extracellular nucleotides: involvement of focal adhesion kinase and phosphatidylinositol 3-kinasemediated pathways. Thromb Haemost 93(4): 735-742

Lee S, Chen TT, Barber CL, Jordan MC, Murdock J, Desai S, Ferrara N, Nagy A, Roos KP, Iruela-Arispe ML (2007) Autocrine VEGF signaling is required for vascular homeostasis. Cell 130(4): 691-703

Okabe-Kado J, Kasukabe T (2003) Physiological and pathological relevance of extracellular NM23/NDP kinases. J Bioenerg Biomembr 35(1): 89-93

Pollard JW (2004) Tumour-educated macrophages promote tumour progression and metastasis. Nat Rev Cancer 4(1): 71 -78

Ralevic V, Burnstock G (1998) Receptors for purines and pyrimidines. Pharmacol Rev 50: $413-492$

Rumjahn SM, Javed MA, Wong N, Law WE, Buxton IL (2007) Purinergic regulation of angiogenesis by human breast carcinoma-secreted nucleoside diphosphate kinase. Br J Cancer 97(10): 1372-1380

Sakurai Y, Ohgimoto K, Kataoka Y, Yoshida N, Shibuya M (2005) Essential role of Flk-1 (VEGF receptor 2) tyrosine residue 1173 in vasculogenesis in mice. Proc Natl Acad Sci USA 102(4): 1076-1081

Satterwhite CM, Farrelly AM, Bradley ME (1999) Chemotactic, mitogenic, and angiogenic actions of UTP on vascular endothelial cells. Am J Physiol 276(3 Part 2): H1091 - H1097

Seye CI, Yu N, Gonzalez FA, Erb L, Weisman GA (2004) The P2Y2 nucleotide receptor mediates vascular cell adhesion molecule-1 expres- sion through interaction with VEGF receptor-2 (KDR/Flk-1). J Biol Chem 279(34): $35679-35686$

Seye CI, Yu N, Jain R, Kong Q, Minor T, Newton J, Erb L, Gonzalez FA, Weisman GA (2003) The P2Y2 nucleotide receptor mediates UTPinduced vascular cell adhesion molecule-1 expression in coronary artery endothelial cells. J Biol Chem 278(27): 24960-24965

Shibuya M (2006) Differential roles of vascular endothelial growth factor receptor-1 and receptor-2 in angiogenesis. J Biochem Mol Biol 39(5): $469-478$

Strawn LM, McMahon G, App H, Schreck R, Kuchler WR, Longhi MP, Hui TH, Tang C, Levitzki A, Gazit A, Chen I, Keri G, Orfi L, Risau W, Flamme I, Ullrich A, Hirth KP, Shawver LK (1996) Flk-1 as a target for tumor growth inhibition. Cancer Res 56(15): 3540-3545

Tanaka N, Kawasaki K, Nejime N, Kubota Y, Nakamura K, Kunitomo M, Takahashi K, Hashimoto M, Shinozuka K (2004) P2Y receptor-mediated $\mathrm{Ca}(2+)$ signaling increases human vascular endothelial cell permeability. J Pharmacol Sci 95(2): 174-180

Tanaka N, Nejime N, Kagota S, Kubota Y, Yudo K, Nakamura K, Kunitomo M, Takahashi K, Hashimoto M, Shinozuka K (2006) ATP participates in the regulation of microvessel permeability. J Pharm Pharmacol 58(4): $481-487$

White N, Burnstock G (2006) P2 receptors and cancer. Trends Pharmacol Sci 27(4): $211-217$ 